\title{
Specialized ommatidia of the polarization-sensitive dorsal rim area in the eye of monarch butterflies have non-functional reflecting tapeta
}

\author{
Thomas Labhart • Franziska Baumann • \\ Gary D. Bernard
}

Received: 6 February 2009 / Accepted: 10 September 2009/Published online: 30 October 2009

(C) The Author(s) 2009. This article is published with open access at Springerlink.com

\begin{abstract}
Many insects exploit sky light polarization for navigation or cruising-course control. The detection of polarized sky light is mediated by the ommatidia of a small specialized part of the compound eye: the dorsal rim area (DRA). We describe the morphology and fine structure of the DRA in monarch butterflies (Danaus plexippus). The DRA consists of approximately 100 ommatidia forming a narrow ribbon along the dorsal eye margin. Each ommatidium contains two types of photoreceptor with mutually orthogonal microvilli orientations occurring in a 2:6 ratio. Within each rhabdomere, the microvilli are well aligned. Rhabdom structure and orientation remain constant at all retinal levels, but the rhabdom profiles, as seen in tangential sections through the DRA, change their orientations in a fan-like fashion from the frontal to the caudal end of the DRA. Whereas these properties (two microvillar orientations per rhabdom, microvillar alignment along rhabdomeres, ommatidial fan array) are typical for insect DRAs in general, we also report and discuss here a novel feature. The ommatidia of monarch butterflies are equipped with reflecting tapeta, which are directly connected to the proximal ends of the rhabdoms. Although tapeta are also present in the DRA, they are separated from the rhabdoms by a space of approximately $55 \mu \mathrm{m}$
\end{abstract}

Electronic supplementary material The online version of this article (doi:10.1007/s00441-009-0886-7) contains supplementary material, which is available to authorized users.

T. Labhart $(\bowtie) \cdot$ F. Baumann

Institute of Zoology, University of Zurich,

Winterthurerstrasse 190,

CH 8057 Zurich, Switzerland

e-mail: labhart@zool.uzh.ch

G. D. Bernard

Department of Electrical Engineering, University of Washington, Seattle, WA 98195-2500, USA effectively inactivating them. This reduces self-screening effects, keeping polarization sensitivity of all photoreceptors of the DRA ommatidia both high and approximately equal.

Keywords Polarization vision · Compound eye · Dorsal rim area $\cdot$ Tapetum $\cdot$ Monarch butterfly, Danaus plexippus (Insecta)

\section{Introduction}

As a result of sunlight scattering in the atmosphere, sky light is partially plane-polarized. Following the rule of firstorder Rayleigh scattering (Strutt 1871), the prevailing oscillation plane (e-vector orientation) is oriented orthogonally to an imaginary straight line connecting an observed point in the sky to the sun. The pattern of polarized sky light, although invisible to man, offers polarizationsensitive organisms a useful reference for visual compass orientation. Insects can exploit polarized sky light for various orientation tasks. They can use it to maintain a straight course as observed in crickets (Brunner and Labhart 1987), locusts (Mappes and Homberg 2004), flies (von Philipsborn and Labhart 1990), and dung beetles (Dacke et al. 2003). Central place foragers such as bees and ants have a polarization compass both to measure and to adjust traveling direction in the context of path integration (Collett and Collett 2000; Labhart and Meyer 2002; Wehner and Srinivasan 2003). Long-range migrators such as locusts (Homberg 2004) and some lepidopterans including monarch butterflies (Reppert et al. 2004) have also been suggested to orient by exploiting sky light polarization during their journeys, although monarchs in another behavioral study failed to respond to e-vector orientation (Stalleicken et al. 2005). 
The detection of polarized sky light in insects is mediated by the ommatidia of just a small part of the compound eye, viz., the dorsal rim area (DRA), which is dedicated exclusively to this task. The ommatidia of the DRA are specialized in ways that make them especially suitable for polarization vision (Labhart and Meyer 1999; Wehner and Labhart 2006). Because of their impressive migrating skills (e.g., Brower 1995, 1996), monarch butterflies have long been objects of keen observation for generations of naturalists. In recent years, since scientists have begun to use experimental approaches under controlled stimulus conditions (Mouritsen and Frost 2002; Reppert et al. 2004; Stalleicken et al. 2005; Sauman et al. 2005), the monarch has become an important model system for studying the physiological mechanisms of insect navigation. After the opsin expression patterns in the retina (Sauman et al. 2005) and the electrophysiology of photoreceptors in the DRA (Stalleicken et al. 2006) have previously been described, we present here a detailed account of the anatomical characteristics of the DRA, the monarch's detector for polarized sky light. In addition to characteristic structural specializations found also in the DRAs of other insects, we have observed a previously unreported optical peculiarity in the monarch. As in most butterflies, the ommatidia in the monarch compound eyes are equipped with reflecting tapeta that create eye glow. Although tapeta are also present in the DRA, they do not produce such eye glow, because they are separated from the rhabdoms by a space of approximately $55 \mu \mathrm{m}$ occupied by the light-insensitive retinula cell R9. This optical inactivation of tapetal reflection reduces self-screening effects, thereby keeping the polarization sensitivity (PS) of all photoreceptors of an ommatidium both high and approximately equal.

\section{Materials and methods}

Pupae of the monarch butterflies (Danaus plexippus) were purchased from London Pupae Supplies (Oxford, UK). After eclosion, the adults were kept at $27^{\circ} \mathrm{C}$ and $70 \%$ relative humidity under long day conditions ( $\mathrm{L} / \mathrm{D}=14 / 10 \mathrm{~h}$ ). Lighting was provided by Osram L20W/10S daylight lamps. For histological examination, the eyes were fixed with $2.5 \%$ glutaraldehyde in $0.05 \mathrm{M} \mathrm{Na}$-cacodylate buffer adjusted to an osmolarity of $350 \mathrm{mOsm}$, for $2 \mathrm{~h}$ at $4^{\circ} \mathrm{C}$. Following post-fixation with $1.3 \% \mathrm{OsO}_{4}$ in $0.2 \mathrm{M} \mathrm{S}$ collidine buffer for $2 \mathrm{~h}$ at room temperature, the tissue was dehydrated with 2,2-dimethoxypropane and embedded in Epon 812. For light microscopy, 1- $\mu \mathrm{m}$ sections were stained with methylene blue and viewed and photographed with a Zeiss Axiophot (Zeiss, Oberkochen, Germany) or an Olympus BX61 (Olympus Optical, Tokyo, Japan). For transmission electron microscopy, silver to gold sections were contrasted with $0.7 \%$ uranyl acetate in water for 10 min and with $2.7 \%$ lead citrate in water for $10 \mathrm{~min}$ and photographed with a Zeiss EM 109 (Zeiss, Oberkochen, Germany). For scanning electron microscopy, mounted monarch heads were gold sputtered and photographed with a Hitachi S4000 (Hitachi, Naka, Japan). Eye glow was studied as explained in detail by Briscoe and Bernard (2005). Tapetal reflectance spectra were calculated by following an analytical procedure (Brekhovskikh 1960; Briscoe et al. 2003) based on the dimensions of tapetal platelets and spaces observed in longitudinal electronmicroscopical sections by using a refractive index of 1.40 for platelets and 1.00 for spaces.

\section{Results}

Regular ommatidia in the dorsal part of the eye

Our findings for the regular ommatidia in the dorsal area (DA) of the eye (Fig. 1a) are consistent with those of Kolb (1985) for another nymphalid species, the small tortoiseshell (Aglais urticae), as pertaining to the structure of the dioptric apparatus, the arrangement of photoreceptor and pigment cells, and a tracheal basket forming a tapetum. Thus, a brief description of the regular ommatidia of the monarch will suffice here. The monarch eye is of the apposition type, i.e., the crystalline cone directly connects to the distal end of the rhabdom. Along almost its whole length, the rhabdom is formed by the microvilli of eight receptor cells (Fig. 2a, d). In ommatidial cross sections, the "vertical" cells (V cells; receptors 1 and 5) are oriented along a dorso-ventral axis of the eye, whereas the "horizontal" cells (H cells; receptors 3 and 7) are horizontally arranged, and the "diagonal" cells (D cells; receptors 2, 4, 6 and 8) have intermediate orientations (cell designation according to Kolb 1985). Near the proximal end of the rhabdom, the $\mathrm{V}$ cells are replaced by cell $\mathrm{R} 9$, which has a curious bi-lobed shape. Rhabdom cross sections exhibit roundish profiles. The length of the rhabdom increases from dorsal to ventral, with 220-230 $\mu \mathrm{m}$ directly adjacent to the DRA, c. $280 \mu \mathrm{m}$ in the 15 th ommatidial row from the dorsal eye margin, and c. $320 \mu \mathrm{m}$ from the 30th row onward. Eight tracheoles run along the proximal part of each ommatidium. Immediately below the rhabdom, they fuse to form the tracheal basket containing an array of platelets, which have the function of a reflecting tapetum. The crystalline cone and the distal retinulae are surrounded by the primary pigment cells, containing large pigment granules. The secondary pigment cells ensheath the retinulae along their whole length and contain small pigment granules at all levels. We have not studied the basal pigment 

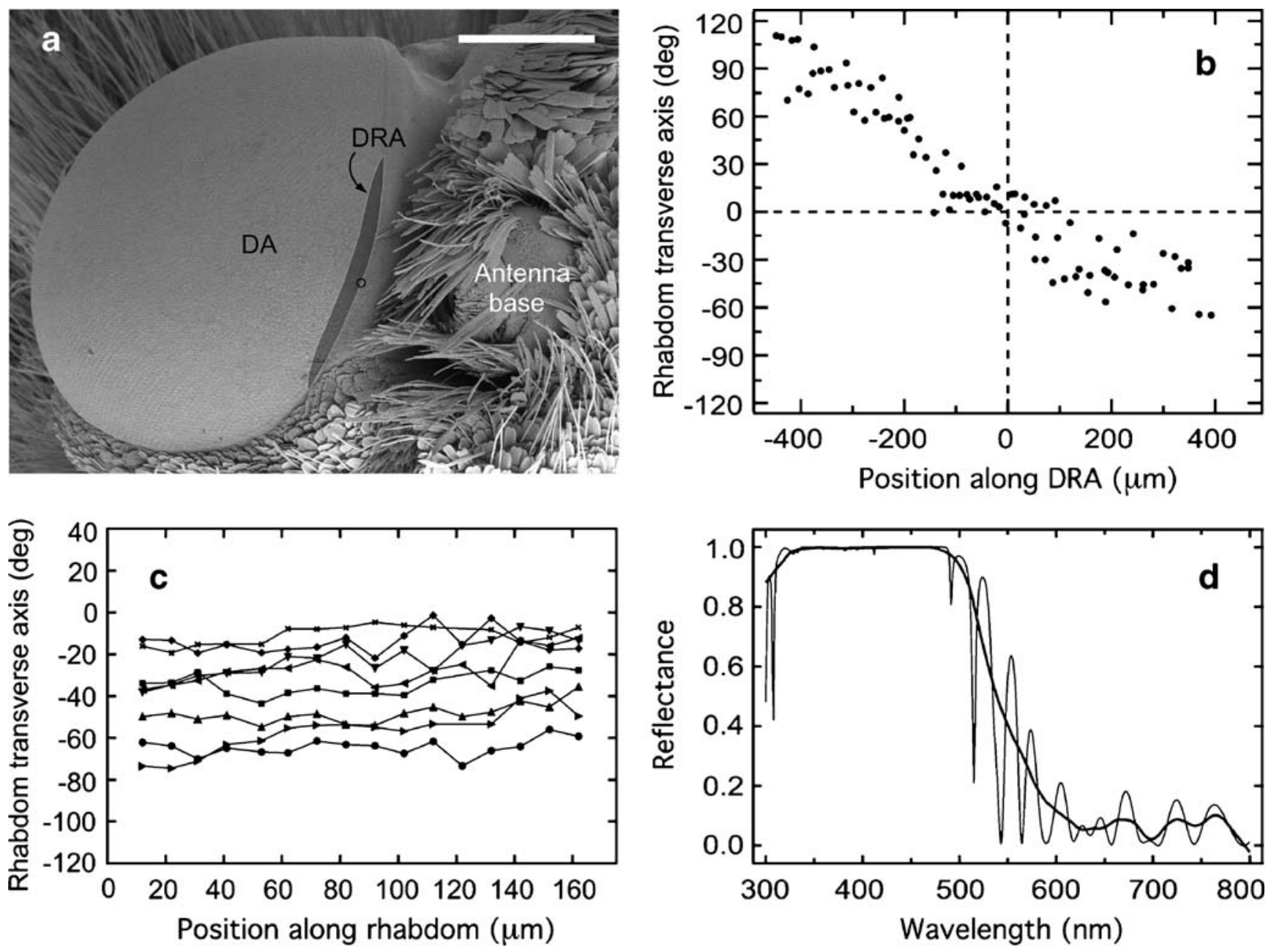

Fig. 1 Characteristics of the dorsal rim area $(D R A)$ of the compound eye. a Position and extension of the DRA. Scanning electron micrograph of the left eye of a monarch butterfly (dorsal and slightly ipsilateral view). The DRA (dark gray area) comprises approximately 100 ommatidia and is $2-3$ ommatidia wide and approximately 45 ommatidia long. The midpoint of the DRA is at the dorsal origin of the horizontal rows of facets (circle), which is located lateral to the antenna base ( $D A$ unspecialized dorsal area). Bar $0.5 \mathrm{~mm}$. b, c Orientation of rhabdom transverse axes (long axes of rectangular rhabdom cross sections). The rhabdom transverse axis indicates the microvilli orientation of the $\mathrm{H}$ receptors (cf. Fig. 2b, e). b Graphic representation of rhabdom transverse axes (ordinate) as a function of position along the DRA (abscissa). Rhabdom transverse axes are relative to the dorsal eye margin $\left(0^{\circ}\right)$, whereas rhabdom position is relative to the center of the DRA $(0 \mu \mathrm{m}$; positive numbers: anterior $)$.

cells. In the photoreceptor cells, pigment density is highest in the distal part. The retinulae are not twisted.

The microvilli arrangement appears irregular (Fig. 2a, d) in ultra-thin cross sections at the electron-microscope level. This agrees with observations made by Kolb (1985) who has found that the microvilli orientation along the rhabdom of individual receptor cells is not constant in serial ultra-thin cross sections of Aglais eyes; in particular, the $\mathrm{V}$ cells regularly change their microvilli orientation

The graph quantifies the observation (Fig. 3) that the rhabdom transverse axes and, thus, the microvilli orientation of the photoreceptors change in a fan-like fashion along the fronto-caudal axis of the DRA. Data are from a single tangential section through the dorsalmost part of the eye ( $185 \mu \mathrm{m}$ below cornea surface) containing most (87) of the approximately 100 ommatidia of the DRA. c Graphic representation of rhabdom transverse axes in the eight identified ommatidia shown in Fig. 2c, f at different retinal levels. Rhabdom transverse axes (ordinate) are relative to the dorsal eye margin $\left(0^{\circ}\right)$, whereas positions along rhabdom (abscissa) are relative to the distal end of the rhabdom $(0 \mu \mathrm{m})$. In each of the eight ommatidia (different symbols), the rhabdom transverse axis is fairly constant indicating that the microvilli are well aligned along the rhabdom. d Reflectance spectrum of a tapetum in the DRA (thin line high resolution, thick line smoothed [Lowess 0.1] spectrum)

along the rhabdom, and the microvilli are often curved (Kolb 1985).

Specialized ommatidia in the DRA of the eye

The ommatidia situated at the dorsal rim of the eye differ markedly in structure and size from those of the dorsal main retina. The DRA is a narrow eye region containing approximately 100 ommatidia (counts in two eyes: 107 and 94); it is 

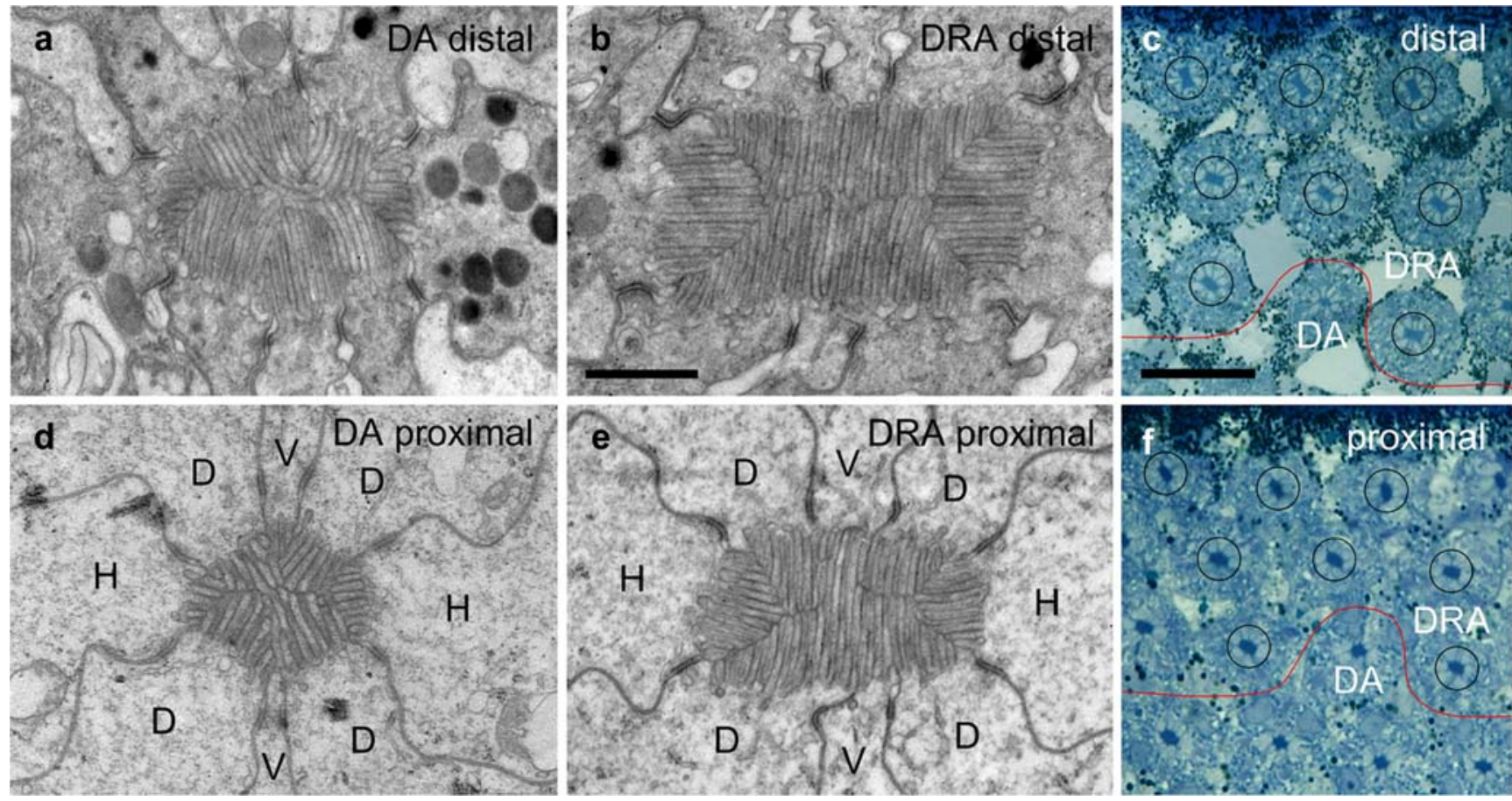

Fig. 2 Rhabdom structure and orientation at various retinal levels in the dorsal rim area $(D R A)$ of the compound eye. a, b, d, e Transmission electron micrographs of rhabdom cross sections in the DRA (b, e) and, for comparison, of the unspecialized dorsal area ( $D A$; $\mathbf{a}, \mathbf{d})$ at distal $(\mathbf{a}, \mathbf{b})$ and proximal levels $(\mathbf{d}, \mathbf{e})$ of the retina $(V$ vertical cells [receptors 1,5$], H$ horizontal cells [receptors 3, 7], $D$ diagonal

cells [receptors 2, 4, 6, 8]). Bar $1 \mu$ m.c, f Light micrographs of eight identified DRA ommatidia (circled) at a distal (c) and a proximal (f) retinal level (red lines border between DRA and unspecialized DA). Note the almost identical orientations of the rhabdom transverse axes (long axes of the rectangular rhabdom cross sections) relative to the eye margin (top) at the two retinal levels. Bar $20 \mu \mathrm{m}$

about 45 ommatidia long (counts: 43,48 ) and two to three ommatidia wide (Figs. 1a, 3). These numbers correspond well with those of a group of ommatidia at the monarch's dorsal eye margin, which exclusively express UV opsin as found by immunohistochemistry (Sauman et al. 2005). In the intact eye, the DRA cannot be recognized directly, but the dorsal origin of the horizontal rows of facets (Fig. 1a, small circle), which is found next to the base of the antenna, serves as a useful landmark for the location of its midpoint.

In tangential sections through the dorsalmost part of the Danaus eye, the rhabdoms of the DRA contrasted with the DA rhabdoms by their conspicuous rectangular shapes and their larger sizes (Fig. 2b, e; see also Fig. 4 in Reppert et al. 2004). Rhabdom sizes varied between individuals. Distally, DRA rhabdoms measured between $1.0 \mu \mathrm{m}$ by $2.5 \mu \mathrm{m}$ to 2.5 $\mu \mathrm{m}$ by $4 \mu \mathrm{m}$ with cross-sectional areas of $2.5 \mu \mathrm{m}^{2}$ to $10 \mu \mathrm{m}^{2}$, whereas DA rhabdoms had areas of about $1.5 \mu \mathrm{m}^{2}$ to $3 \mu \mathrm{m}^{2}$. In a given eye, the areas of DRA rhabdoms were always larger (twice to three times) than those of the DA rhabdoms. However, the rhabdoms in the DRA were significantly shorter $(140 \mu \mathrm{m}$ to $160 \mu \mathrm{m})$ than those of the DA $(220 \mu \mathrm{m}$ to 320 $\mu \mathrm{m}$, see above). Thus, the rhabdoms of DRA ommatidia were both shorter and wider than those of the DA. We estimate that rhabdom volumes were roughly the same in both eye regions.

Along its whole length, the rhabdom is formed by the microvilli of eight photoreceptors (Fig. 2b, e). In contrast to the DA, the arrangement of the microvilli in DRA rhabdoms exhibits a strict order. Electron micrographs of cross sections at different positions along the rhabdom always show just two approximately orthogonal microvilli orientations. The two $\mathrm{H}$ cells sit at the narrow sides of the rectangular rhabdom profile forming microvilli, which are approximately orthogonal to those of the other six photoreceptors (mean $\pm \mathrm{SD}: 89.5^{\circ} \pm 7.2^{\circ}, n=56$ ). Microvilli orientations are closely correlated with the orientation of the rhabdom profile. Thus, in order to estimate microvillar alignment along the rhabdomeres, we measured the long axes of rhabdom profiles (rhabdom transverse axes) in a group of eight identified rhabdoms in light-microscopic sections at different (10-15) retinal levels. As demonstrated in Figs. 1c, 2c, f, rhabdom orientations remained fairly constant at all levels. The standard deviations of the rhabdom transverse axes in the eight ommatidia ranged from $4.2^{\circ}$ to $10.6^{\circ}$ (mean: $\pm 5.9^{\circ}$ ) indicating that the microvilli of individual receptor cells were well aligned.

Although rhabdom orientation within an ommatidium is constant, the orientation of the rhabdom profiles as seen in tangential sections of the DRA varies in a fan-like fashion (Figs. 1b, 3d). In the center of the DRA, the long axes of 

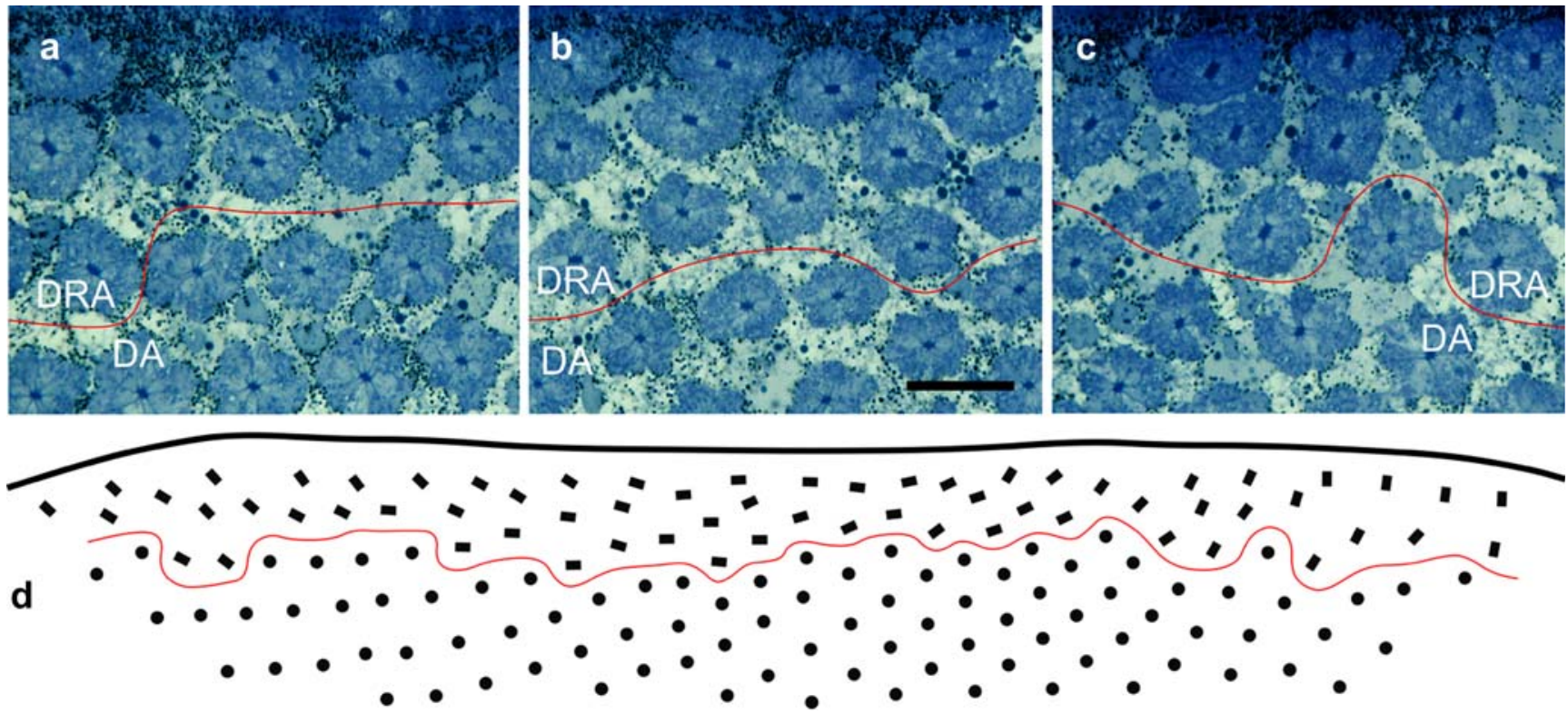

Fig. 3 Ommatidial arrangement and rhabdom orientation as seen in a tangential section of the dorsal rim area $(D R A)$ of the compound eye (DA unspecialized dorsal area). a-c Light micrographs of the midfrontal, middle, and mid-caudal part of the DRA, respectively (red lines border between the DRA and DA). Note the different orientations of the rhabdom transverse axes (long axes of the rectangular rhabdom cross sections) relative to the eye margin (top).
Bar $20 \mu \mathrm{m}$. d Represention of ommatidium position and orientation (same section as in a-c; left anterior). The section contains 64 crosssections of DRA ommatidia (rectangles). Because of the eye curvature, the rest of the approximately 100 DRA rhabdoms are not contained in this section (dots unspecialized dorsal ommatidia, red line as in $\mathbf{a}-\mathbf{c}$ )

proximal Aglais DRA, 10 receptor cell profiles per ommatidium are visible, whereas in the Danaus DRA, more than eight receptor cell profiles have never been observed. However, in Aglais, the sub-rhabdomeral position of the nucleus of cell 9 (see Fig. 2 in Kolb 1985) also suggests a separation of rhabdom end and tapetum.

Apart from the peculiar position of the tapetum, both light-microscopic long and cross sections suggest no obvious differences in the optical properties of the DA and DRA ommatidia of Danaus. The corneal lenses and crystalline cones are well developed, the crystalline cone directly connects to the rhabdom, and the degree of pigmentation is similar in the ommatidia of the DRA and the adjacent DA. tapetum appears proximally to R9 only (Fig. 4 b, e), i.e., the tapetum is separated from the end of the rhabdom by a space of approximately $55 \mu \mathrm{m}$, which is occupied by R9. In longitudinal sections of the tapeta at the electronmicroscopic level, the DA and DRA reveal no noticeable differences, and the spacing and thickness of the platelets indicates that the tapeta of both eye regions are well suited for reflecting both UV and visible light to at least $480 \mathrm{~nm}$ (Fig. 1d).

The morphology of R9 in the Danaus DRA differs from that in the Aglais DRA, where the two-limbed cell 9 appears between cells 5 and 6 and between cells 1 and 8, forming a few microvilli (Kolb 1986). Thus, in the

\section{Discussion}

Structure and function of the dorsal rim ommatidia

The ommatidia in the DRA of the monarch butterfly show the typical features of insect ommatidia associated with polarized sky light detection, such as wide and short rhabdoms, two types of photoreceptor with mutually orthogonal microvilli orientation and well-aligned microvilli in each receptor (for a review, see Labhart and Meyer 1999). Microvillar alignment in combination with short 

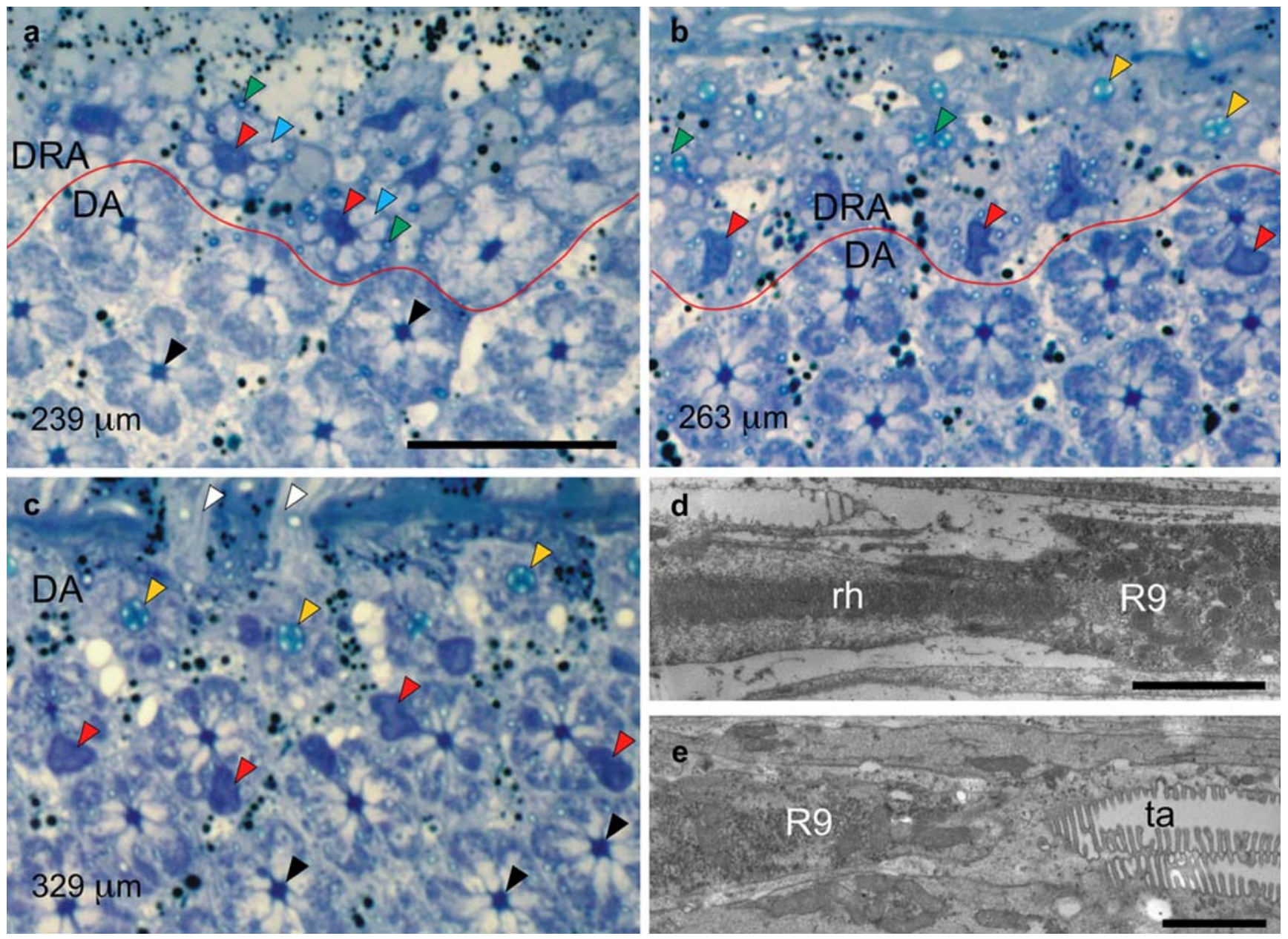

Fig. 4 a-c Position of proximal retinula cell R9 with respect to rhabdom and tapetum in the ommatidia of the dorsal rim area $(D R A)$ and unspecialized dorsal area $(D A)$ of the compound eye. Light micrographs of tangential sections through the dorsalmost part of an eye at progressively deeper proximal levels of the retina. Specifications in micrometers (bottom left) indicate section levels relative to cornea surface. R9 cells can be recognized by their dark blue stain (red lines border between DRA and unspecialized DA). Bar $20 \mu \mathrm{m}$ a Section plane just proximal to the end of the rhabdoms in the DRA. R9 cells (red arrowheads, large dark profiles) in the DRA are surrounded by the axons of $\mathrm{V}, \mathrm{H}$, and $\mathrm{D}$ cells (blue arrowheads, large light profiles) and tracheoles (green arrowheads, small profiles), replacing the rhabdoms. In the longer DA ommatidia, V, H, and D cells still form rhabdoms at this level (black arrowheads). b Section plane near the junction between R9 cells and tapeta in the DRA. DRA ommatidia exhibit either R9 (red arrowheads, large dark profiles), tapeta (yellow arrowheads), or tracheoles in the process of fusing (green arrowheads). In one of the DA retinulae, the proximal end of

rhabdoms indicates that the photoreceptors are strongly polarization-sensitive, whereas the irregular microvilli arrangement in the DA suggests low PS (Nilsson et al. 1987). As demonstrated by electrophysiological recordings, these expectations are, indeed, met. The monarch DRA is monochromatic and contains exclusively UV receptors (Stalleicken et al. 2006; Sauman et al. 2005). The average the rhabdom is indicated by the presence of an eccentric R9 (red arrowhead). c Section plane near the junction between rhabdoms and tapeta in the DA. At this level, the axons of the DRA ommatidia have left the retina, and only DA retinulae are visible. Since ommatidial length increases with distance from the eye rim, this section shows DA ommatidia at three levels: (1) V, H, and D cells form the rhabdoms (black arrowheads), (2) H, D, and the bilobed R9 cells (red arrowheads, dark profiles) form the rhabdoms, and (3) tapeta (yellow arrowheads). Retinula cell axons (white arrowheads) penetrate the basement membrane. d, e Position of R9 with respect to rhabdom and tapetum in the ommatidia of the DRA. Electron micrographs of longitudinal sections in the proximal part of the retina (left distal). Bars $2 \mu \mathrm{m}$ d The elongated microvilli-less cell R9 begins at the proximal end of the rhabdom $(r h)$. e The reflecting tapetum $(t a)$ begins at the proximal end of R9. The proximal end of the rhabdom is immediately followed by the tapetum in the DA, whereas R9 sits between the rhabdom and the tapetum in the DRA

PS of these cells has been found to be 9.4 (Stalleicken et al. 2006). The DA is trichromatic and contains UV, blue, and green receptors (Sauman et al. 2005; Stalleicken et al. 2005). PS values have been assessed for UV and green cells: they average 2.9 and 2.8, respectively (Stalleicken et al. 2005).

The orientation of the rhabdom profiles changes in a fanlike fashion from the frontal to the caudal end of the DRA 
indicating a corresponding change of microvilli orientation and, thus, of the e-vector tuning axes of the photoreceptors. Similar ommatidial fan arrangements have been found in the DRAs of many other insects (e.g., desert ants and honey bees: Wehner 1982; flies: Strausfeld and Wunderer 1985; crickets: Blum and Labhart 2000; locusts: Homberg and Paech 2002; two scarabaeid beetles: Labhart et al. 1992; Dacke et al. 2003) including the saturniid moth Antherea polyphemus (Anton-Erxleben and Langer 1988). The fan array in the DRA seems to reflect the general ommatidial geometry of the compound eye, and a special developmental program for shaping the array might not be necessary. The fan array provides a set of first-order polarization analyzers (photoreceptors) with different e-vector tuning orientations. As demonstrated in crickets, the optic lobe contains three types of polarization-sensitive neurons (POL-neurons), which are tuned to different e-vector orientations. Each type of POL-neuron receives input from a large number of ommatidia with appropriate orientations (Labhart et al. 2001). Different e-vector orientations elicit specific combinations of activities among the three tuning types of POL-neuron providing a triplet code for e-vector orientation, which is evaluated in the central brain (Sakura et al. 2008).

Each ommatidium of the DRA contains two types of photoreceptor with mutually orthogonal microvilli (H cells vs. V and D cells). The presence of two orthogonal microvillar types of photoreceptor per ommatidium is a hallmark of the insect DRA, in general (Labhart and Meyer 1999; Wehner and Labhart 2006). It provides the basis of polarization antagonism as demonstrated in POL-neurons of several insects (Labhart and Meyer 2002; Wehner and Labhart 2006). Polarization antagonism effectively enhances e-vector contrast, allowing the system to operate even under low degrees of celestial polarization (Labhart 1996, 1999).

Our histological assessment of the dioptric system (lens, crystalline cone), rhabdom structure, and pigment distribution of DRA ommatidia does not suggest any major differences in the optics of the DA and the DRA. Indeed, the visual fields of monarch photoreceptors exhibit the typical bell shapes in both eye regions, and the acceptance angles are only slightly larger in the DRA $\left(4.0^{\circ}\right)$ than in the DA $\left(2.9^{\circ}\right.$; Stalleicken et al. 2005); this might be a result of the different rhabdom sizes. Although the visual fields of the ommatidia are similar in the DA and the DRA of the monarch, they are considerably enlarged in the DRA in many other insects. In these insects, the optics of the DRA is specialized in ways that strongly increase the acceptance angles of the polarization-sensitive photoreceptors. The cornea can contain light-scattering structures (honey bees: Meyer and Labhart 1981; other hymenopterans: Aepli et al. 1985; cockchafers: Labhart et al. 1992), the screening pigment or the tracheal sheath, which normally shield the ommatidia from each other, can be missing or reduced (crickets: Burghause 1979; locusts:
Homberg and Paech 2002), or a mismatch can occur between focal length and rhabdom position (cockchafers: Labhart et al. 1992; crickets: Ukhanov et al. 1996). Increased visual fields in the DRA have been interpreted as an adaptation to the perception of polarized light under partly cloudy sky conditions. Integration over a large area of sky reduces the effect of irregularities within the pattern of polarized sky light caused by clouds (Labhart 1999). However, enlarged visual fields seem not to be essential for polarized sky light navigation. For instance, in the desert ant Cataglyphis, which uses the pattern of polarized sky light as a key compass cue during extended foraging trips (e.g., Wehner 1997; Wehner and Müller 2008), the visual field size of the DRA receptors $\left(5.4^{\circ}\right.$; Labhart 1986$)$ is in the same range as that found in the monarch.

\section{Non-functional tapeta in the DRA}

In one remarkable aspect, the optics of the ommatidia of the DRA and of the main retina differ significantly. As in most butterflies, the ommatidia of monarchs are equipped with a tapetum. When the eyes of monarch butterflies are observed under incident illumination coaxial with the observer's direction of view, they exhibit a characteristic eye glow (Miller and Bernard 1968). Incident light, which has traveled through the rhabdom without being absorbed, is reflected by the tapetum. Eye glow is the fraction of light also escaping absorption on its way back through the rhabdom. Interestingly, no eye glow can be observed in the ommatidia of the DRA. At best, a weak and diffuse bluish gleam between the facets can be distinguished (present study; Stalleicken et al. 2006). The monarch DRA is UVmonochromatic (Sauman et al. 2005; Stalleicken et al. 2006) and, thus, the tapetum might be adapted to reflect UV only, such that the glow becomes inperceptible on visual inspection. However, model calculations of the reflection spectrum show that the DRA tapeta reflect both UV and visible light (Fig. 1d; for details, see Electronic Supplementary Material Fig. 1S, Table 1S). We therefore favor the idea that the missing glow is a result of the unusual position of the tapeta in the DRA. Whereas the proximal end of the rhabdom directly abuts the tapetum in the main retina, rhabdom and tapetum are separated by a space of $55 \mu \mathrm{m}$, which is held by retinula cell R9, in the DRA. Light propagating down the rhabdom waveguide is confined, but once the light reaches the proximal end of the rhabdom, waveguiding ceases, and the light spreads into the basal region, its intensitiy decreasing rapidly with distance. Thus, only a small fraction of the light leaving the rhabdom reaches the tapetum, and only another small fraction of the reflected light will reach the rhabdom once again, and so the light leaving the eye is too weak to be properly observed and to be physiologically effective. 
What could be the reason that the tapetum has been uncoupled from the rhabdom in the monarch DRA? In night-active insects, the function of a tapetum consists in doubling the light path in the receptor for more light absorption, i.e., for higher sensitivity (Miller 1979). In many day-active butterflies, screening pigment selectively absorbing certain spectral bands affects the spectral tuning of photoreceptors; this has been proposed to enhance color discrimination. The tapetum is thought both to assist both in further shaping spectral sensitivity and to make up for the overall loss of quanta by reducing spectral bandwidth (Stavenga 2002; Arikawa et al. 1999). Why has the tapetum become non-functional in the monarch DRA? Several reasons can be proposed. (1) Fine-tuning the spectral sensitivity of the photoreceptors makes no sense in the monochromatic monarch DRA. (2) The state of polarization might be disturbed in light reflected by the tapetum. However, as measured in the butterfly Heteronympha, this effect seems to be weak (Nilsson and Howard 1989). (3) In photoreceptors with long rhabdoms, PS can be significantly reduced because of self-screening effects (Nilsson et al. 1987). Short rhabdoms, therefore, favor PS since selfscreening is minimized. As in other insects, the photoreceptors in the monarch DRA are significantly shorter than in the main retina $(140-160 \mu \mathrm{m}$ vs. up to $320 \mu \mathrm{m}$ in the main retina). Reflection of the light arriving at the proximal end of the rhabdom back into the rhabdom would effectively double rhabdom length and thus decrease PS, even if the polarization state remained unaffected by the tapetal reflection. However, the rhabdom contains not just one but two mutually orthogonal microvilli orientations that could reduce the effect of self-screening to some degree (Nilsson et al. 1987). Nevertheless, the $\mathrm{H}$ cells with horizontally oriented microvilli contribute much less to the rhabdom volume than the $\mathrm{V}$ and $\mathrm{D}$ cells with their vertical microvilli (Fig. 2b, e). In this case, the PS values of the photoreceptors are expected to diverge more, the longer the rhabdom: PS will increase in the $\mathrm{H}$ cells and decrease in the V and D cells (Nilsson et al. 1987). Strong asymmetry of input may disturb the proper operation of polarizationopponent POL-neurons, since their e-vector response functions become highly asymmetric (see Fig. 6 in Nilsson et al. 1987). Thus, the maintenance of a short effective rhabdom length by removal of tapetal reflection keeps the PS of all photoreceptors at a high and approximately equal level.

How much does the optical inactivation of the tapetum influence PS of the DRA photoreceptors? If the DRA tapetum were moved to the usual position at the basal tip of the rhabdom, would its reflected light be substantial enough to have a negative impact on PS of DRA receptors? Nilsson and Howard (1989) have measured the relative intensity of eyeshine at the level of the corneal facets. We have used their data from Aglais urticae and their assumption of $80 \%$ excitation efficiency to estimate the light intensity at the basal end of the rhabdom in the monarch. Reflected intensity in both modes measured at the cornea is $4.61 \%$. Rhabdom length is $320 \mu \mathrm{m}$ (Kolb 1985) such that 0.8 $\mathrm{T}^{2}=0.0461$, where $\mathrm{T}$ is the one-way transmission coefficient of the rhabdom at $\lambda_{\max }$. Thus, $\mathrm{T}=24 \%$, and consequently, absorbance is $\log 0.24 / 320 \mu \mathrm{m}=0.0019 \mu \mathrm{m}^{-1}$. Nilsson and Howard (1989) conclude that the photopigments absorb half the light per $120 \mu \mathrm{m}$ of rhabdom resulting in a somewhat higher absorbance of $0.0025 \mu^{-1}$. For Vanessa cardui, the optical density of the rhabdom is 0.62 (Bernard 1983) for $400 \mu \mathrm{m}$ rhabdoms (Briscoe et al. 2003) so that absorbance is $0.0016 \mu^{-1}$. Thus, for the purposes of this discussion, we assume an absorbance of $0.002 \mu^{-1}$ for the rhabdoms of the monarch DRA. The transmittance of regular Aglais rhabdoms at $\lambda_{\max }$ is $24 \%$ but is $50 \%$ for monarch DRA rhabdoms because they are only $150 \mu \mathrm{m}$ long. Since the tapetum is a perfect reflector at $\lambda_{\max }$ (Fig. 1d), all of that light is reflected, propagating back up the rhabdom where again $50 \%$ of it is absorbed. Therefore, a tapetum would increase the absorbed fraction of incident light from $50 \%$ to $75 \%$. The DRA tapetum, however, is optically inactive because of its separation from the rhabdom tip.

To quantify the effect of tapetum inactivation on PS, we have created a computational model for the two situations, i.e., with and without a tapetum, under the following assumptions. The DRA rhabdom is a waveguide, in which light intensity decreases with increasing depth because of absorption by visual pigment. The tapetum reflects $100 \%$ of the incident light, the fraction of the rhabdom occupied by vertical microvilli is 0.706 as measured in electronmicroscopic cross sections, the microvillar dichroic ratio $d r$ is 10 , and the absorbance is $0.002 \mu \mathrm{m}^{-1}$ at $\lambda_{\max }$ (for full details, see Electronic Supplementary Material, Text S1). We calculated the amount of monochromatic light absorbed by the rhabdom fractions occupied by vertical and horizontal microvilli, respectively, each for vertically and horizontally polarized light. All values were computed for both $150 \mu \mathrm{m}$ and $300 \mu \mathrm{m}$ long rhabdoms representing the situations without and with a tapetum (for full details, see Electronic Supplementary Material, Text S1). The ratio of absorption for polarized light oscillating parallel and orthogonal to the microvillar axes, respectively, indicates the PS value of photoreceptors with vertically and horizontally oriented microvilli $\left(\mathrm{PS}_{\mathrm{vert}}, \mathrm{PS}_{\text {hor }}\right)$. For the tapetal case, $\mathrm{PS}_{\mathrm{vert}}=8.0$ and $\mathrm{PS}_{\mathrm{hor}}=12.5$, and without a tapetum, the corresponding values are 8.9 and 11.2. Thus, although a tapetum would reduce $\mathrm{PS}_{\mathrm{vert}}$ by only about $10 \%$, the difference between PS $_{\text {vert }}$ and PS hor $_{\text {would significantly }}$ but not dramatically be increased from 2.3 to 4.5 . 


\section{Concluding remarks}

The ommatidia of the DRA in monarch butterflies show the typical structural specializations found in other polarizationsensitive insect species (Labhart and Meyer 1999; Wehner and Labhart 2006). In addition, the monarch DRA is monochromatic, and the photoreceptors are strongly polarization-sensitive (Sauman et al. 2005; Stalleicken et al. 2006). This leaves little doubt that monarch butterflies can exploit sky light polarization for orientation. However, behavioral tests of polarization sensitivity have proven controversial (Reppert et al. 2004; Stalleicken et al. 2005), and it seems that the exploitation of skylight polarization is strongly context dependent.

Acknowledgements We are grateful to Dr. Eric P. Meyer for critical comments on the manuscript.

Open Access This article is distributed under the terms of the Creative Commons Attribution Noncommercial License which permits any noncommercial use, distribution, and reproduction in any medium, provided the original author(s) and source are credited.

\section{References}

Aepli F, Labhart T, Meyer EP (1985) Structural specializations of the cornea and retina at the dorsal rim of the compound eye in hymenopteran insects. Cell Tissue Res 239:19-24

Anton-Erxleben F, Langer H (1988) Functional morphology of the ommatidia in the compound eye of the moth, Antheraea polyphemus (Insecta, Saturniidae). Cell Tissue Res 252:385-396

Arikawa K, Mizuno S, Scholten DGW, Kinoshita K, Seki T, Kitamoto J, Stavenga DG (1999) An ultraviolet absorbing pigment causes a narrow-band violet receptor and a single-peaked green receptor in the eye of the butterfly Papilio. Vision Res 39:1-8

Bernard GD (1983) Dark-processes following photoconversion of butterfly rhodopsins. Biophys Struct Mech 9:277-286

Blum M, Labhart T (2000) Photoreceptor visual fields, ommatidial array and receptor axon projections in the polarisation-sensitive dorsal rim area of the cricket compound eye. J Comp Physiol [A] 186:119-128

Brekhovskikh LM (1960) Waves in layered media. Academic Press, New York, pp 56-61

Briscoe AD, Bernard GD (2005) Eyeshine and spectral tuning of long wavelength-sensitive rhodopsins: no evidence for red-sensitive photoreceptors among five Nymphalini butterfly species. J Exp Biol 208:687-698

Briscoe AD, Bernard GD, Szeto AS, Nagy LM, White RH (2003) Not all butterfly eyes are created equal: rhodopsin absorption spectra, molecular identification, and localization of ultraviolet-, blue-, and green-sensitive rhodopsin-encoding mRNAs in the retina of Vanessa cardui. J Comp Neurol 458:334-349

Brower LP (1995) Understanding and misunderstanding the migration of the monarch butterfly (Nymphalidae) in North America: 1857-1995. J Lepid Soc 49:304-385

Brower LP (1996) Monarch butterfly orientation: missing pieces of a magnificent puzzle. J Exp Biol 199:93-103

Brunner D, Labhart T (1987) Behavioural evidence for polarization vision in crickets. Physiol Entomol 12:1-10
Burghause FMHR (1979) Die strukturelle Spezialisierung des dorsalen Augenteils der Grillen (Orthoptera, Grylloidea). Zool Jb Physiol 83:502-525

Collett M, Collett T (2000) How do insects use path integration for their navigation? Biol Cybern 83:245-259

Dacke M, Nordström P, Scholtz CH (2003) Twilight orientation to polarised light in the crepuscular dung beetle Scarbaeus zambesianus. J Exp Biol 260:1535-1543

Homberg U (2004) In search of the sky compass in the insect brain. Naturwissenschaften 91:199-208

Homberg U, Paech A (2002) Ultrastructure and orientation of ommatidia in the dorsal rim area of the locust compound eye. Arthropod Struct Dev 30:271-280

Kolb G (1985) Ultrastructure and adaptation in the retina of Aglais urticae (Lepidoptera). Zoomorphology 105:90-98

Kolb G (1986) Retinal ultrastructure in the dorsal rim and large dorsal area of the eye of Aglais urticae (Lepidoptera). Zoomorphology 106:244-246

Labhart T (1986) The electrophysiology of photoreceptors in different eye regions of the desert ant, Cataglyphis bicolor. J Comp Physiol [A] 158:1-7

Labhart T (1996) How polarization-sensitive interneurons of crickets perform at low degrees of polarization. J Exp Biol 199:1467-1475

Labhart T (1999) How polarization-sensitive interneurons of crickets see the polarization pattern of the sky: a field study with an optoelectronic model neuron. J Exp Biol 202:757-770

Labhart T, Meyer EP (1999) Detectors for polarized skylight in insects: a survey of ommatidial specializations in the dorsal rim area of the compound eye. Microsc Res Tech 47:368-379

Labhart T, Meyer EP (2002) Neural mechanisms in insect navigation: polarization compass and odometer. Cur Opin Neurobiol 12:707-714

Labhart T, Meyer EP, Schenker L (1992) Specialized ommatidia for polarization vision in the compound eye of cockchafers, Melolontha melolontha (Coleoptera, Scarabaeidae). Cell Tissue Res 268:419-429

Labhart T, Petzold J, Helbling H (2001) Spatial integration in polarization-sensitive interneurones of crickets: a survey of evidence, mechanisms and benefits. J Exp Biol 204:2423-2430

Mappes M, Homberg U (2004) Behavioral analysis of polarization vision in tethered flying locusts. J Comp Physiol [A] 190:61-68

Meyer EP, Labhart T (1981) Pore canals in the cornea of a functionally specialized area of the honey bee's compound eye. Cell Tissue Res 216:491-501

Miller WH (1979) Ocular optical filtering. In: Autrum H (ed) Handbook of sensory physiology VII/6A. Springer, Berlin Heidelberg New York, pp 69-143

Miller WH, Bernard GD (1968) Butterfly glow. J Ultrastruct Res 24:286-294

Mouritsen H, Frost BJ (2002) Virtual migration in tethered flying monarch butterflies reveals their orientation mechanisms. Proc Natl Acad Sci USA 99:10162-10166

Nilsson D-E, Howard J (1989) Intensity and polarization of the eyeshine in butterflies. J Comp Physiol [A] 166:51-56

Nilsson D-E, Labhart T, Meyer E (1987) Photoreceptor design and optical properties affecting polarization sensitivity in ants and crickets. J Comp Physiol [A] 161:645-658

Philipsborn A von, Labhart T (1990) A behavioral study of polarization vision in the fly, Musca domestica. J Comp Physiol [A] 167:737-743

Reppert SM, Zhu H, White RH (2004) Polarized light helps monarch butterflies navigate. Curr Biol 14:155-158

Sakura M, Lambrinos T, Labhart T (2008) Polarized skylight navigation in insects: model and electrophysiology of e-vector coding by neurons in the central complex. J Neurophysiol 99:667-682 
Sauman I, Briscoe AD, Zhu HS, Shi DD, Froy O, Stalleicken J, Yuan Q, Casselman A, Reppert SM (2005) Connecting the navigational clock to sun compass input in monarch butterfly brain. Neuron 46:457-467

Stalleicken J, Mukhida M, Labhart T, Wehner R, Frost B, Mouritsen H (2005) Do monarch butterflies use polarized skylight for migratory orientation? J Exp Biol 208:2399-2408

Stalleicken J, Labhart T, Mouritsen H (2006) Physiological characterization of the compound eye in monarch butterflies with focus on the dorsal rim area. J Comp Physiol [A] 192:321-331

Stavenga DG (2002) Reflections on colourful ommatidia of butterfly eyes. J Exp Biol 205:1077-1085

Strausfeld NJ, Wunderer H (1985) Optic lobe projections of marginal ommatidia in Calliphora erythrocephala specialized for detecting polarized light. Cell Tissue Res 242:163-178

Strutt J (Lord Rayleigh) (1871) On the light from the sky, its polarization and colour. Phil Mag 41:107-120
Ukhanov K, Leertouwer HL, Gribakin FG, Stavenga DG (1996) Dioptrics of the facet lenses in the dorsal rim area of the cricket Gryllus bimaculatus. J Comp Physiol [A] 179:545-552

Wehner R (1982) Himmelsnavigation bei Insekten. Neurophysiologie und Verhalten. Neujahrsbl Naturforsch Ges Zürich 184:1-132

Wehner R (1997) The ant's celestial compass system: spectral and polarization channels. In: Lehrer $M$ (ed) Orientation and communication in arthropods. Birkhäuser, Basel, pp 145-185

Wehner R, Labhart T (2006) Polarization vision. In: Warrant EJ, Nilsson D-E (eds) Invertebrate vision. Cambridge University Press, Cambridge, pp 291-348

Wehner R, Müller M (2008) The significance of direct sunlight and polarized skylight in the ant's celestial system of navigation. Proc Natl Acad Sci USA 103:12575-12579

Wehner R, Srinivasan MV (2003) Path integration in insects. In: Jeffery KJ (ed) The neurobiology of spatial behavior. Oxford University Press, Oxford, pp 9-30 